\title{
Sun Stroke
}

National Cancer Institute

\section{Source}

National Cancer Institute. Sun Stroke. NCI Thesaurus. Code C35047.

A reaction to prolonged exposure to high temperatures that can result in a failure of the body's temperature control system. It is often characterized by body temperature over 105 degrees, dehydration, headache, dizziness, nausea, vomiting, and muscle weakness. 